\title{
Do Human Rationales Improve Machine Explanations?
}

\author{
Julia Strout, Ye Zhang and Raymond J. Mooney \\ Department of Computer Science \\ University of Texas at Austin \\ \{jstrout, yezhang, mooney\}@cs.utexas.edu
}

\begin{abstract}
Work on "learning with rationales" shows that humans providing explanations to a machine learning system can improve the system's predictive accuracy. However, this work has not been connected to work in "explainable AI" which concerns machines explaining their reasoning to humans. In this work, we show that learning with rationales can also improve the quality of the machine's explanations as evaluated by human judges. Specifically, we present experiments showing that, for $\mathrm{CNN}$ based text classification, explanations generated using "supervised attention" are judged superior to explanations generated using normal unsupervised attention.
\end{abstract}

\section{Introduction}

Recently, the need for explainable artificial intelligence (XAI) has become a major concern due to the increased use of machine learning in automated decision making (Gunning, 2017; Aha, 2018). On the other hand, work on "learning with rationales" (Zaidan et al., 2007; Zhang et al., 2016) has shown that humans providing explanatory information supporting their supervised classification labels can improve the accuracy of machine learning. These human annotations that can explain classification labels are called rationales. In particular, for text categorization, humans select phrases or sentences from a document that most support their decision as rationales.

However, there is no work connecting "learning from rationales" with improving XAI, although they are clearly complementary problems.

Contribution We explore whether learning from human explanations actually improves a system's ability to explain its decisions to human users. Specifically, we show that for explanations for text classification in the form of selected passages that best support a decision, training on hu- man rationales improves the quality of a system's explanations as judged by human evaluators.

Attention mechanisms (Bahdanau et al., 2015) have become standard practice in computer vision and text classification (Vaswani et al., 2017; Yang et al., 2016). In both computer vision and textbased tasks, learned attention weights have been shown through human evaluation to be useful explanations for a model's decisions (Park et al., 2018; Rocktäschel et al., 2015; Hermann et al., 2015; Xu et al., 2015); however, attention's explanatory power has come into question in recent work (Jain and Wallace, 2019), which we discuss in Section 2.

Traditional attention mechanisms are unsupervised; however, recent work has shown that supervising attention with human annotated rationales can improve learning for text classification based on Convolutional Neural Networks (CNNs) (Zhang et al., 2016). While this work alludes to improved explainability using supervised attention, it does not explicitly evaluate this claim. We extend this work by evaluating whether supervised attention using human rationales, rather than unsupervised attention, actually improves explanation. Explanations from both models are full sentences that the model has weighted as being most important to the document's final classification.

While automated evaluations of explanations (e.g. comparing them to human gold-standard explanations (Lei et al., 2016)) can be somewhat useful, we argue that because the goal of machine explanations is to help users, they should be directly evaluated by human judges. Machine explanations can be different from human ones, but still provide good justification for a decision (Das et al., 2017). This opinion is shared by other researchers in the area (Doshi-Velez, 2017), but human evaluation is often avoided due to the time required and difficulty of conducting human trials. We believe it is a 
necessary element of explainability research, and in this work, we compare the explanations from the two models through human evaluation on Mechanical Turk and find that the model trained with human rationales is judged to generate explanations that better support its decisions.

\section{Related Work}

There is a growing body of research on explainable AI (Koh and Liang, 2017; Ribeiro et al., 2016; Li et al., 2016; Hendricks et al., 2018), but it is not connected to work on learning with human rationales, which we review below.

As discussed above, Zhang et al. (2016) demonstrate increased predictive accuracy of CNN models augmented with human rationales. Here, we first reproduce their predictive results, and then focus on extracting and evaluating explanations from the models. Lei et al. (2016) present a model that extracts rationales for predictions without training on rationales. They compare their extracted rationales to human gold-standard ones through automated evaluations, i.e., precision and recall. Bao et al. (2018) extend this work by learning a mapping from the human rationales to continuous attention. They transfer this mapping to low resource target domains as an auxiliary training signal to improve classification accuracy in the target domain. They compare their learned attention with human rationales by calculating their cosine distance to the 'oracle' attention.

None of the above related work asks human users to evaluate the generated explanations. However, Nguyen (2018) does compare human and automatic evaluations of explanations. That work finds that human evaluation is moderately, but statistically significantly, correlated with the automatic metrics. However, it does not evaluate any explanations based on attention, nor do the explanations make use of any extra human supervision.

As mentioned above, there has also been some recent criticism of using attention as explanation (Jain and Wallace, 2019), due to a lack of correlation between the attention weights and gradient based methods which are more "faithful" to the model's reasoning. However, attention weights offer some insight into at least one point of internal representation in the model, and they also impact the training of the later features. Our contribution is to measure how useful these attention based explanations are to humans in understand- ing a model's decision as compared to a different model architecture that explicitly learns to predict which sentences make good explanations.

In this work, we have human judges evaluate both attention based machine explanations and machine explanations trained from human rationales, thus connecting learning from human explanations and machine explanations to humans.

\section{Models and Dataset}

\subsection{Models}

We replicate the work of Zhang et al. (2016) and use a $\mathrm{CNN}$ as our underlying baseline model for document classification. To model a document, each sentence is encoded as a sentence vector using a $\mathrm{CNN}$, and then the document vector is formed by summing over the sentence vectors. We use two variations of this baseline model, a rationale-augmented CNN $(\boldsymbol{R A}-\boldsymbol{C N N})$ and an attention based CNN (AT-CNN) (Yang et al., 2016). RA-CNN is trained on both the document label and the rationale labels. In this model, the document vector is a weighted sum of the composite sentence vectors, where the weight is the probability of the sentence being a rationale. In AT-CNN, the document vector is still a weighted sum of sentence $\mathrm{CNN}$ vectors, but the weight is not learned from rationales. Rather, a trainable context vector is introduced from scratch. We calculate the interaction between this context vector and each sentence vector to induce attention weights over the sentences. The only difference between RA$\mathrm{CNN}$ and AT-CNN is that RA-CNN relies on the human annotated rationales to learn the sentence weight at training time, while AT-CNN learns the sentence weight without utilizing any human rationales. For the details of these two models and training see Zhang et al. (2016).

\subsection{Explanations}

At test time, each model can provide explanations for its classification decision by either choosing the sentences with the largest probability of being a rationale in RA-CNN or the sentences with the largest attention weights in AT-CNN. By comparing the quality of explanations output by the two models at test time, we can judge whether capitalizing on human explanations at training time can improve the machine explanations at test time. 


\subsection{Dataset}

We evaluate the explanations for both models on the movie review dataset from Zaidan et al. (2007). It contains 1,000 positive reviews and 1,000 negative reviews where 900 of each are annotated with human rationales. Each review is a document consisting of 32 sentences on average, and each annotated document contains about 8 rationale sentences. We use the 1,800 annotated documents as the training set, and the remaining 200 documents without extra annotation as test. The human rationales are used as supervision in RA-CNN but not in AT-CNN.

\subsection{Classification Accuracy}

The classification accuracy of each model on the test set is summarized in Table 1. Since there is variance across multiple trials, we pick the best performing model across several trials for human evaluation of the explanations.

Table 1 reproduces Zhang et al. (2016)'s finding that providing human explanations to machines at training time (RA-CNN) improves predictive accuracy compared to learning explanations without human annotations (AT-CNN). Our results differ slightly from theirs in that our AT-CNN also outperforms the baseline Doc-CNN. We attribute this difference to possible slight variations in our implementation of AT-CNN.

Note there are other works on learning attention that could potentially increase the prediction accuracy (Lin et al., 2017; Devlin et al., 2018), but none of them are directly comparable to RA-CNN. We introduced the smallest difference (whether the sentence vector is trained using the rationale label) between AT-CNN and RA-CNN to make a fair comparison between their generated explanations.

The focus of this work is on evaluating explanations rather than predictive accuracy, so we turn our attention to the question: Does humans explaining themselves to machines improve machines explaining themselves to humans? We will explore this in the next section.

\section{Explanation Evaluation Methods}

We use Amazon Mechanical Turk (AMT) to evaluate the explanations from both AT-CNN and RACNN.

\begin{tabular}{ccc} 
Doc-CNN & AT-CNN & RA-CNN \\
\hline $86.00 \%$ & $88.50 \%$ & $90.00 \%$
\end{tabular}

Table 1: Classification accuracy for movie reviews.

\subsection{HIT Design}

Our Human Intelligence Task (HIT) shows a worker two copies of a test document along with the document's classification. Each copy of the document has a subset of sentences highlighted as explanations for the final classification. This subset is chosen as the 3 sentences with the largest weights from either AT-CNN's attention weights or RA-CNN's supervised weighting. We also evaluated a baseline model that selects 3 sentences at random. Given two randomly ordered documents, a worker must choose which document's highlighted sentences best support the overall classification. If the worker determines that both are equally supportive (or not supportive), then they can select 'equal'. We only show workers documents that were correctly classified by both models. This resulted in 166 documents from the 200 in the test set. An example from one HIT is in Appendix A.

\subsection{Quality Control}

In an effort to receive quality results from the crowd, we employ two strategies from crowdsourcing research: gold standard questions and majority voting (Hsueh et al., 2009; Eickhoff and de Vries, 2013). Gold standard questions are designed to weed out unreliable workers who either do not understand the goal of the task or are poor workers. If a worker gets the gold standard question wrong, then we assume that their other responses are untrustworthy and do not use them.

We also employ majority voting, which requires that at least two workers who pass the gold standard question agree on an answer. For greater than $90 \%$ of the test documents, a majority vote was found after having three workers perform the task. Less than $10 \%$ of the test documents required a fourth worker who passed the gold question to break a tie. We also chose to require the 'Master' qualification that AMT uses to designate the best workers on the platform.

\section{Explanation Evaluation Results}

Table 3 contains the results for comparing the top 3 explanations from AT-CNN to the top 3 explanations from RA-CNN for the 166 test documents 


\begin{tabular}{|c|c|c|c|}
\hline Label & Rank & AT-CNN & RA-CNN \\
\hline \multirow{3}{*}{ Pos } & 1 & $\begin{array}{l}\text { archer is also bound by the limits of new york soci- } \\
\text { ety, which is as intrusive as any other in the world. }\end{array}$ & the performances are absolutely breathtaking. \\
\hline & 2 & $\begin{array}{l}\text { the marriage is one which will unite two very pres- } \\
\text { tigious families, in a society where nothing is more } \\
\text { important than the opinions of others . }\end{array}$ & $\begin{array}{l}\text { there are a few deft touches of filmmaking that are } \\
\text { simply outstanding, and joanne woodward' narra- } \\
\text { tion is exquisite. }\end{array}$ \\
\hline & 3 & $\begin{array}{l}\text { the supporting cast is also wonderful, with several } \\
\text { characters so singular that they are indelible in one's } \\
\text { memory. }\end{array}$ & $\begin{array}{l}\text { the supporting cast is also wonderful, with several } \\
\text { characters so singular that they are indelible in one's } \\
\text { memory. }\end{array}$ \\
\hline \multirow{3}{*}{ Neg } & 1 & $\begin{array}{l}\text { soon the three guys are dealing dope to raise funds } \\
\text {, while avoiding the cops and rival dealer sampson } \\
\text { simpson (clarence williams iii). }\end{array}$ & $\begin{array}{l}\text { it's just that the comic setups are obvious and the } \\
\text { payoffs nearly all fall flat. }\end{array}$ \\
\hline & 2 & $\begin{array}{l}\text { only williams stands out (while still performing on } \\
\text { the level of his humor-free comedy rocket man), } \\
\text { but that is because he's imprisoned throughout most } \\
\text { of the film, giving a much needed change of pace } \\
\text { (but mostly swapping one set of obvious gags for } \\
\text { another). }\end{array}$ & $\begin{array}{l}\text { watching the film clean and sober, you are bound } \\
\text { to recognize how truly awful it is . }\end{array}$ \\
\hline & 3 & $\begin{array}{l}\text { watching the film clean and sober, you are bound } \\
\text { to recognize how truly awful it is . }\end{array}$ & $\begin{array}{l}\text { the film would have been better off by sticking with } \\
\text { the " rebel" tone it so eagerly tries to claim. }\end{array}$ \\
\hline
\end{tabular}

Table 2: Top 3 explanations from both models for both a positive and negative correctly classified test document.

\begin{tabular}{c|c|c} 
RA-CNN & AT-CNN & Equal \\
\hline $43.47 \%$ & $20.48 \%$ & $36.14 \%$
\end{tabular}

Table 3: AMT results comparing explanations from RA-CNN to AT-CNN. Workers were asked to choose which document's highlighted sentences were a better explanation for the final classification.

\begin{tabular}{c|c|c} 
AT-CNN & Random & Equal \\
\hline $57.23 \%$ & $15.66 \%$ & $27.12 \%$
\end{tabular}

Table 4: AMT results comparing AT-CNN to the random baseline.

where the models each correctly classified the document. The statistics presented are the percentage of times reliable workers agreed that one model's explanations better supported the document's classification or were equal.

Overall, it is clear that RA-CNN is providing better explanations for the plurality of test documents $(43.47 \%)$. The explanations are considered equal $36.14 \%$ of the time, and the remaining $20.48 \%$ of the documents were better explained by AT-CNN.

After seeing these results, we decided to run another baseline test to ensure that AT-CNN explanations are reasonable and can at least beat a weak baseline. The results from comparing ATCNN explanations to randomly sampled sentences from the test document are in Table 4. From these results we can see that AT-CNN is beating the random baseline the majority of the time, demonstrating that attention, even without human supervision, can provide helpful explanations for a model's decision.

To further understand the differences between the explanations from AT-CNN and RA-CNN, we calculated statistics to find the amount of overlap in the top three explanatory sentences from each model. In $33.5 \%$ of the test documents, the models share no explanation sentences, in $43.1 \%$ they share one explanation sentence, in $22.2 \%$ they share two explanation sentences, and they share all three in $1.2 \%$. When considering just the most highly weighted sentence, or top explanation, the models agree $18.6 \%$ of the time. So while it is relatively rare for the models to produce the same top explanatory sentence, we chose to show humans three explanatory sentences per test document to provide insight even in those matching cases.

Table 2 contains the top 3 explanations from each model for two test documents. In both examples, AT-CNN extracts sentences that are more plot related and give less insight into the reviewer's opinion as compared to RA-CNN. These sentences are generally less helpful for understanding the classification of the movie review. In the second example, both models have identified a good explanatory sentence: "watching the film clean and sober, you are bound to recognize how truly awful it is." However, AT-CNN ranks it as less important than two sentences that primarily describe the plot of the film while RA-CNN only ranks another, equally explanatory sentence as more important.

An interesting future avenue for evaluation is to compare explanations from when the models 
make incorrect predictions. We found a trend in the explanations for test documents that both models misclassified where RA-CNN produced explanations that supported the misclassification while AT-CNN produced more explanations that supported the correct classification, despite the model's decision. While this analysis is too small scale to be conclusive, this raises the question for future work: Do we want our explanation systems to offer the best support for the chosen decision or would it be more beneficial if they provide an explanation that brings the decision into question?

\section{Conclusion}

This paper has demonstrated that training with human rationales improves explanations for a model's classification decisions as evaluated by human judges. We show that while an unsupervised attention based model does provide some valuable explanations, as proven in the experiments comparing to a random baseline, a supervised attention model that trains on human rationales outperforms those results.

\section{Acknowledgements}

This research was supported by the DARPA XAI program through a grant from AFRL. The views and conclusions contained herein are those of the authors and should not be interpreted as necessarily representing the official policies or endorsements, either expressed or implied, of the U.S. Government.

\section{References}

David Aha, editor. 2018. Proceedings of the IJCAI Workshop on Explainable Artificial Intelligence. Melbourne, Australia.

Dzmitry Bahdanau, Kyunghyun Cho, and Yoshua Bengio. 2015. Neural machine translation by jointly learning to align and translate. International Conference on Learning Representations.

Yujia Bao, Shiyu Chang, Mo Yu, and Regina Barzilay. 2018. Deriving machine attention from human rationales. In Conference on Empirical Methods in Natural Language Processing.

Abhishek Das, Harsh Agrawal, Larry Zitnick, Devi Parikh, and Dhruv Batra. 2017. Human attention in visual question answering: Do humans and deep networks look at the same regions? Computer Vision and Image Understanding, 163:90-100.
Jacob Devlin, Ming-Wei Chang, Kenton Lee, and Kristina Toutanova. 2018. Bert: Pre-training of deep bidirectional transformers for language understanding. arXiv preprint arXiv:1810.04805.

Been Doshi-Velez, Finale; Kim. 2017. Towards a rigorous science of interpretable machine learning. In Spring Series on Challenges in Machine Learning: "Explainable and Interpretable Models in Computer Vision and Machine Learning”.

Carsten Eickhoff and Arjen P de Vries. 2013. Increasing cheat robustness of crowdsourcing tasks. Information retrieval, 16(2):121-137.

David Gunning. 2017. Explainable artificial intelligence (XAI). Defense Advanced Research Projects Agency (DARPA).

Lisa Anne Hendricks, Ronghang Hu, Trevor Darrell, and Zeynep Akata. 2018. Grounding visual explanations. In European Conference of Computer Vision $(E C C V)$.

Karl Moritz Hermann, Tomas Kocisky, Edward Grefenstette, Lasse Espeholt, Will Kay, Mustafa Suleyman, and Phil Blunsom. 2015. Teaching machines to read and comprehend. In Advances in $\mathrm{Neu}$ ral Information Processing Systems, pages 16931701.

Pei-Yun Hsueh, Prem Melville, and Vikas Sindhwani. 2009. Data quality from crowdsourcing: A study of annotation selection criteria. In Proceedings of the NAACL HLT 2009 Workshop on Active Learning for Natural Language Processing, HLT '09, pages 2735, Stroudsburg, PA, USA. Association for Computational Linguistics.

Sarthak Jain and Byron C. Wallace. 2019. Attention is not explanation. In Proceedings of the 2019 North American Chapter of the Association for Computational Linguistics (NAACL).

Pang Wei Koh and Percy Liang. 2017. Understanding black-box predictions via influence functions. In 34th International Conference on Machine Learning.

Tao Lei, Regina Barzilay, and Tommi Jaakkola. 2016. Rationalizing neural predictions. In Conference on Empirical Methods in Natural Language Processing.

Jiwei Li, Xinlei Chen, Eduard Hovy, and Dan Jurafsky. 2016. Visualizing and understanding neural models in NLP. In Proceedings of NAACL-HLT, pages 681691.

Zhouhan Lin, Minwei Feng, Cicero Nogueira dos Santos, Mo Yu, Bing Xiang, Bowen Zhou, and Yoshua Bengio. 2017. A structured self-attentive sentence embedding. In International Conference on Learning Representations. 
Dong Nguyen. 2018. Comparing automatic and human evaluation of local explanations for text classification. In Proceedings of the 2018 Conference of the North American Chapter of the Association for Computational Linguistics: Human Language Technologies, Volume 1 (Long Papers), pages 10691078.

Dong Huk Park, Lisa Anne Hendricks, Zeynep Akata, Anna Rohrbach, Bernt Schiele, Trevor Darrell, and Marcus Rohrbach. 2018. Multimodal explanations: Justifying decisions and pointing to the evidence. In 31st IEEE Conference on Computer Vision and Pattern Recognition.

Marco Tulio Ribeiro, Sameer Singh, and Carlos Guestrin. 2016. Why should i trust you?: Explaining the predictions of any classifier. In Proceedings of the 22nd ACM SIGKDD international conference on knowledge discovery and data mining, pages 1135-1144. ACM.

Tim Rocktäschel, Edward Grefenstette, Karl Moritz Hermann, Tomáš Kočiskỳ, and Phil Blunsom. 2015. Reasoning about entailment with neural attention. arXiv preprint arXiv:1509.06664.

Ashish Vaswani, Noam Shazeer, Niki Parmar, Jakob Uszkoreit, Llion Jones, Aidan N Gomez, Łukasz Kaiser, and Illia Polosukhin. 2017. Attention is all you need. In Advances in Neural Information Processing Systems, pages 5998-6008.

Kelvin Xu, Jimmy Ba, Ryan Kiros, Kyunghyun Cho, Aaron Courville, Ruslan Salakhudinov, Rich Zemel, and Yoshua Bengio. 2015. Show, attend and tell: Neural image caption generation with visual attention. In International conference on machine learning, pages 2048-2057.

Zichao Yang, Diyi Yang, Chris Dyer, Xiaodong He, Alex Smola, and Eduard Hovy. 2016. Hierarchical attention networks for document classification. In Proceedings of the 2016 Conference of the North American Chapter of the Association for Computational Linguistics: Human Language Technologies, pages 1480-1489.

Omar Zaidan, Jason Eisner, and Christine Piatko. 2007. Using annotator rationales to improve machine learning for text categorization. In Human Language Technologies 2007: The Conference of the North American Chapter of the Association for Computational Linguistics; Proceedings of the Main Conference, pages 260-267.

Ye Zhang, Iain Marshall, and Byron C Wallace. 2016. Rationale-augmented convolutional neural networks for text classification. In Proceedings of the Conference on Empirical Methods in Natural Language Processing., volume 2016, page 795. NIH Public Access. 


\section{A Sample HIT}

\section{Instructions}

Choose the document where the highlighted text best supports the document's classification.

- Documents $A$ and $B$ are the same movie review, but with different highlighted sentences.

- You should choose the document where the highlighted text best explains the movie's classification.

- A positive review indicates that the author of the review considered it to be a high quality movie, while a negative review indicates

that the reviewer did not.

- If the different documents' highlights seem equally informative you should select 'Equal'.

- Include a brief explanation for why you chose your answer.

\section{Classification: Positive}

\section{Document A}

Robert redford's a river runs through it is not a film i watch often. It is a masterpiece -- one of the better films of recent years. Until 1994, it was my second favorite film of all time. The acting and direction is top-notch -- never sappy, always touching. A friend of mine once reported that he avoided it because "i was afraid it would just be really politically correct, and tick me off. All i could do was tell him to go in unbiased, and enjoy. It is one of the few movies that has completely reduced me to tears. But certain memories should not often be rereleased -- in the last few shots, you have to cry. Upon my first viewing i left bawling. It is not flawless -- but it is so very good, that you can't help but be effected. The opening is dangerously nolstalgic and sentimental -- watching these shots of people who have been dead so long, gives you a feeling of perspective and history observation that you will find in very few other films. Martin scorsese once described the movies as a dream state -- like taking dope, and immersing yourself in an alternative world. That is what a river runs through it does. It exploits the unique power of cinema to engross you and help to forget your real self. Both times i've seen it, its been hard ( again to quote scorsese) waking up. But the dream is lovely.

\section{Document B}

Robert redford's a river runs through it is not a film i watch often. It is a masterpiece -- one of the better films of recent years. Until 1994, it was my second favorite film of all time. The acting and direction is top-notch -- never sappy, always touching. A friend of mine once reported that he avoided it because "i was afraid it would just be really politically correct, and tick me off. All i could do was tell him to go in unbiased, and enjoy. It is one of the few movies that has completely reduced me to tears. But certain memories should not often be rereleased -- in the last few shots, you have to cry. Upon my first viewing i left bawling. It is not flawless -- but it is so very good, that you can't help but be effected. The opening is dangerously nolstalgic and sentimental -- watching these shots of people who have been dead so long, gives you a feeling of perspective and history observation that you will find in very few other films. Martin scorsese once described the movies as a dream state -- like taking dope, and immersing yourself in an alternative world. That is what a river runs through it does. It exploits the unique power of cinema to engross you and help to forget your real self. Both times i've seen it, its been hard (again to quote scorsese) waking up. But the dream is lovely.

Which document's highlights better support the classification?

Document A

Document B

Equal

Explain your choice:

Figure 1: A sample HIT asking workers to compare two explanations for the same movie review. 